\title{
A Reconfigurable, Multi-Gigahertz Pulse Shaping Circuit Based on Distributed Transversal Filters
}

\author{
Yunliang Zhu*, Jonathan D. Zuegel ${ }^{\dagger}$, John R. Marciante ${ }^{\dagger}$, and Hui $\mathrm{Wu}^{*}$ \\ * Department of Electrical and Computer Engineering, University of Rochester, Rochester, NY \\ ${ }^{\dagger}$ Laboratory for Laser Energetics, University of Rochester, Rochester, NY
}

\begin{abstract}
A distributed transversal filter (DTF) is a good candidate for ultrafast pulse shaping in wideband systems like UWB. This paper presents the circuit analysis of DTFs based on transmission line theory. We also show the detailed design procedure of DTFs. A 5-tap prototype DTF is designed and fabricated using microwave PCB substrate and pHEMT discrete transistors. Simulation and initial measurement results demonstrate its pulse shaping capability.
\end{abstract}

\section{INTRODUCTION}

Ultrafast pulse shaping is important in many applications. For example, in wireline communications, pre-emphasis and equalization are used to modify the signal waveform in order to combat dispersion due to bandwidth limitation. Recently, ultra-wideband (UWB) communications has generated new interests in sub-nanosecond pulse generation and processing. In a high-rate, direct-sequence UWB transceiver [1] or a lowrate impulse-radio UWB system [2], pulse shaping is critical to satisfy the stringent spectral and temporal constraints on transmitted signals. The pulse shaping circuit in these wideband systems, however, is difficult to design and implement because of the large bandwidth and complex spectrum mask. Using a number of (discrete) high-Q band-stop filters is undesirable because of the large signal loss, high cost and lack of reconfigurability. Digital programmable FIR filters like those in fiber-optic communication systems usually demand large chip area in an advanced process technology, and consume hundreds of milliwatts power due to the circuit complexity. Apparently they are not suitable for most UWB systems, which are envisioned as inexpensive, low-power, portable devices.

Recent research demonstrated that a distributed transversal filter (DTF) can be a good candidate for multi-gigahertz signal processing such as equalization in $10 \mathrm{~Gb} / \mathrm{s}$ fiber-optic systems [3][4] and sub-nanosecond pulse synthesis[5] . A DTF [6][7] is essentially an analog transversal filter based on distributed amplifiers [8], which are widely used in microwave communication and radar systems because of their unique wideband characteristics. DTFs are also reconfigurable by controlling the gain from each cell[6]. Therefore, it is attractive to further study this architecture in the pulse shaping applications in wideband systems such as UWB. Our goal in this paper is to investigate the pulse shaping capability of a reconfigurable DTF using a discrete prototype.

\section{Distributed TRAnsVers Al Filter}

Fig. 1 shows a distributed transversal filter. The input signal travels along the input transmission line (gate line), is tapped

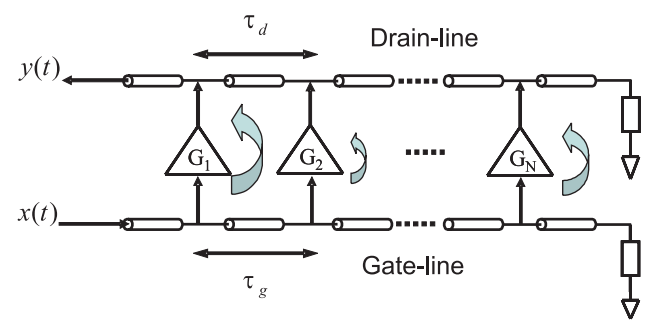

Fig. 1. A distributed transversal filter.

by each gain cell in sequence (from left to right in Fig. 1), and amplified by each cell by a gain proportional to the filter coefficient. The output signals from all gain cells are power-combined on the output transmission line (drain line). Both periodically loaded transmission lines also act as delay elements. The parasitic capacitance of gain cells becomes part of the transmission lines like in a distributed amplifier, which is why DTFs can achieve gigahertz bandwidth.

On the system level, a DTF operates like an FIR filter. The time domain description of the circuit is

$$
y(t)=\sum_{n=1}^{N} G_{n} x\left(t-\sum_{i=0}^{n-1} \tau_{i}\right)
$$

where $G_{n}$ is the gain of gain cell $n$, and $\tau_{i}$ is the delay between gain cell $i$ and $i+1$. Note that $\tau_{i}$ consists of that from both the loaded gate and drain lines, i.e., $\tau_{i}=\tau_{g i}+\tau_{d i}$. The corresponding frequency response is given by

$$
H(\omega)=\sum_{n=1}^{N} G_{n} \exp \left(-j \omega \sum_{i=0}^{n-1} \tau_{i}\right)
$$

Assuming a uniform tap delay $\tau \equiv \tau_{g}=\tau_{d}$

$$
H(\omega)=\sum_{n=1}^{N} G_{n} e^{-j 2 n \omega \tau}
$$

\section{ANALYSIS}

The DTF can be modeled as in Fig. 2. The gate drain lines are loaded by the input and output capacitance of gain cells, respectively. ${ }^{1}$ It is evident that the design of a DTF largely involves that of the loaded gate and drain lines, which determine the DTF's bandwidth and each tap's transfer functions. Circuit analysis of periodically loaded transmission

\footnotetext{
${ }^{1}$ The input and output resistance can be easily included in a more detailed analysis by using a complex impedance.
} 

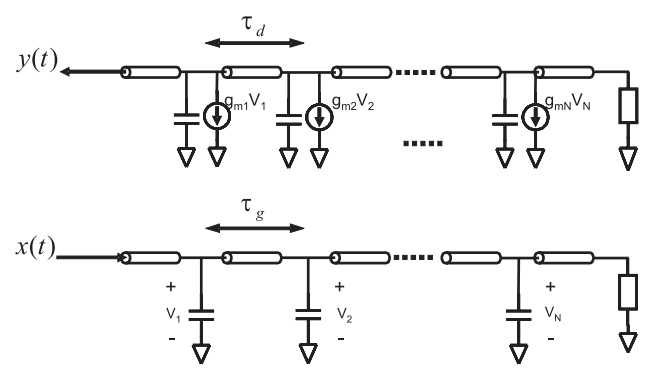

Fig. 2. Circuit model for a DTF.
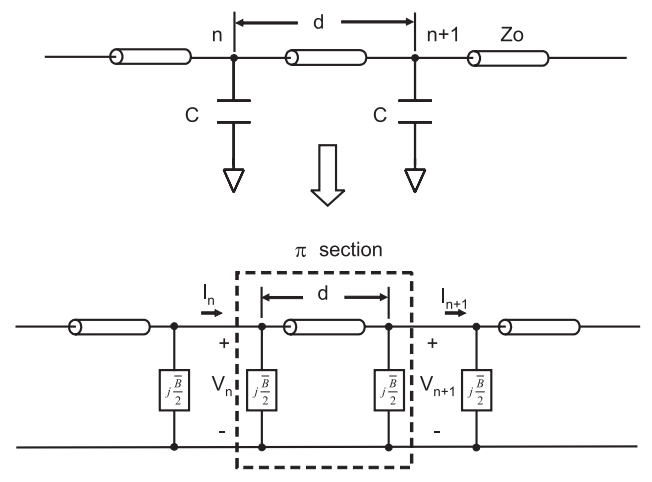

Fig. 3. Periodically loaded transmission line.

lines [9][10] is used here. The drain line uses $\pi$ section as the basic iteration structure, as shown in Fig. 3. Each $\pi$ section consists of a lossless transmission line with length $d$ and two shunt susceptance from the capacitance. The susceptance $\frac{\bar{B}}{2}$ is normalized to the characteristic impedance of unloaded transmission line, $Z_{0}$. We can relate the voltages and currents on two sides of the $n$th $\pi$ section by a $A B C D$ matrix

$$
\left[\begin{array}{l}
V_{n} \\
I_{n}
\end{array}\right]=\left[\begin{array}{ll}
A & B \\
C & D
\end{array}\right]\left[\begin{array}{l}
V_{n+1} \\
I_{n+1}
\end{array}\right]
$$

In its normalized form,

$$
\left[\begin{array}{ll}
A & B \\
C & D
\end{array}\right]=\left[\begin{array}{cc}
\cos \theta-\frac{\bar{B}}{2} \sin \theta & j \sin \theta \\
j \bar{B} \cos \theta+j \sin \theta-j\left(\frac{\bar{B}}{2}\right)^{2} \sin \theta & \cos \theta-\frac{\bar{B}}{2} \sin \theta
\end{array}\right]
$$

where $\theta=\mathrm{kd}$, and $k$ is the propagation constant of the unloaded line.

This periodic structure is capable of supporting a propagating wave. Let $\gamma$ to be the propagation constant of loaded transmission line, i.e., $V_{n+1}=e^{-\gamma d} V_{n}, I_{n+1}=e^{-\gamma d} I_{n}$. By solving the eigenvalue equation of the ABCD matrix, we can find the solution for $\gamma$

$$
\cosh (\gamma d)=\cos \theta-\frac{\bar{B}}{2} \sin \theta
$$

In the passband, the right side of Eqn. 6 should satisfy,

$$
\left|\cos \theta-\frac{\bar{B}}{2} \sin \theta\right|<1
$$

We can also find the characteristic impedance of the load transmission line (Bloch impedance) to be

$$
Z_{B}=Z_{0} \sqrt{\frac{\sin \theta}{\bar{B} \cos \theta+\left(1-\frac{\bar{B}^{2}}{4}\right) \sin \theta}}
$$

The derived $\gamma$ and $Z_{B}$ is also true for T sections [9], which can model the gate line. Noting that there is only a half $T$ section at the input port, the first tap voltage on the gate line is

$$
V_{1}=h_{1, i n} V_{i n}=\left(D_{1}-\frac{B_{1}}{Z_{B}}\right) V_{i n}
$$

where $h_{1, i n}$ is the voltage transfer function from input to the first tap on the gate line, and

$$
\begin{aligned}
D_{1} & =\cos \frac{\theta}{2} \\
B_{1} & =j \sin \frac{\theta}{2}
\end{aligned}
$$

Then the voltage at the $n$th tap on the gate-line is

$$
V_{n}=h_{n, i n} V_{i n}=h_{1, i n} e^{-(n-1) \gamma d} V_{i n}
$$

which generates the current $g_{m n} V_{n}$ on the drain-line. Half of the current is delivered to the output port through $n \pi$ sections of drain line, which gives another $e^{-n \gamma d}$ factor. The other half current is absorbed by the termination. Therefore, under the assumption that both gate and drain line are terminated with $Z_{B}$, the voltage transfer function of the $n$th tap in a DTF is

$$
H_{n}(\omega)=\frac{1}{2} h_{1, i n} e^{-(2 n-1) \gamma d} g_{m n} Z_{B}
$$

where $\gamma, Z_{B}$ and $h_{1, i n}$ is from Eqn. (6), (8) and (9), respectively. Therefore, the overall transfer function of the DTF is

$$
H(\omega)=\frac{1}{2} h_{1, i n} Z_{B} \sum_{n=1}^{N} g_{m n} e^{-(2 n-1) \gamma d}
$$

\section{Circuit Design}

The system specification of the discrete prototype is determined as following: the delay per tap $\tau$ is $100 \mathrm{ps,} \mathrm{which}$ means the sampling frequency $f_{s}$ is $10 \mathrm{GHz}$; the number of taps is set to 5, which is sufficient for pulse shaping in impulseradio UWB systems [11]; the cut-off frequency of both loaded transmission lines are specified to be $7 \mathrm{GHz}$, considering the limitation of PCB implementation; and the Bloch impedance is set to $50 \Omega$.

Based on the bandwidth specification, a discrete transistor, Agilent ATF36077, is chosen for the gain cell. It is a pHEMT device with a $0.2 \mu \mathrm{m}$ gate length, $200 \mu \mathrm{m}$ gate width, packaged in a surface-mountable ceramic package. The device is characterized in frequency domain using a network analyzer. Transmission-reflection-line (TRL) calibration is used to shift the reference plane to the package edge. From measured s-parameters, small-signal equivalent circuit models are extracted at various bias conditions for the initial design (Fig. 4). A Statz model of the transistor provided by the manufacturer is then used in design optimization.

The gain cell is designed as a cascode amplifier (Fig. 5) in order to increase its output impedance and thus reduce the 


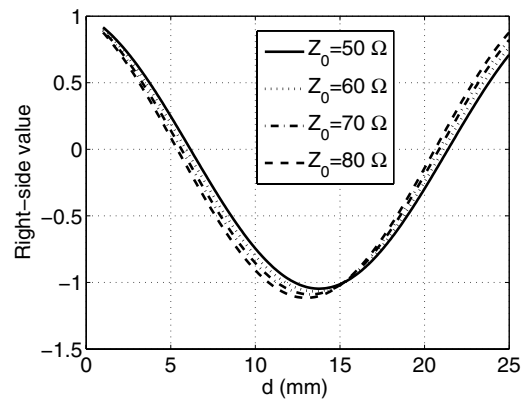

(a)

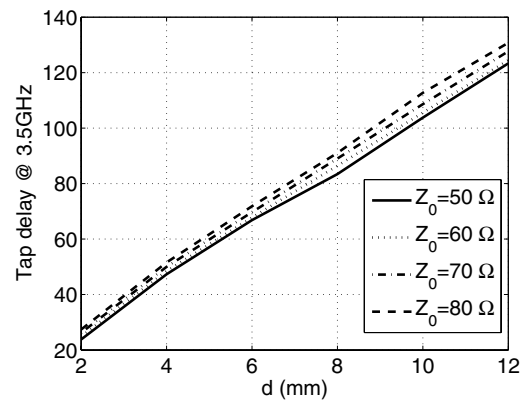

(b)

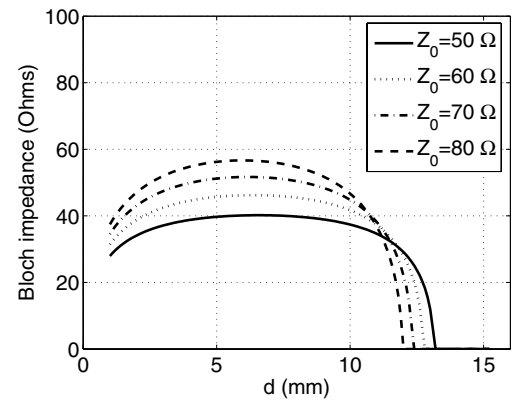

(c)

Fig. 6. Numerical analysis to determine tap spacing $\mathrm{d}$ and unloaded impedance $Z_{0}$.

loss on the drain line. It also helps to reduce parasitic coupling between gate and drain lines. The transistor has a large input capacitance of $0.8 \mathrm{pF}$. So a $0.2 \mathrm{pF}$ input coupling capacitor $C_{c}$ is added to reduce the capacitive loading on the gate line with sacrifice in gain. $C_{c}$ also enables tuning the dc gate voltage of $M_{1}$ in each cell and thus the gain independently. $C_{g}$ provides ac ground to $M_{2}$ gate, and a de-Q resistor $R_{g}$ is added to guarantee stability of the amplifier. They are also fine-tuned independently in each cell to achieve best frequency responses.

Microstrip transmission lines are used to construct the gate and drain lines. The design parameters are the characteristic impedance of the unloaded lines $\left(Z_{0}\right)$ and tap spacing $(d)$. They have to be determined together from the specifications of cut-off frequency $(7 \mathrm{GHz})$, Bloch impedance $(50 \Omega)$ and tap delay (100 ps). The cut-off frequency is evaluated using Eqn. (7) when sweeping $Z_{0}$ from $50 \Omega$ to $80 \Omega$ (Fig. 6a). It is found that $d$ cannot be longer than $11 \mathrm{~mm}$. Then $d$ is set to $9.2 \mathrm{~mm}$ according to the tap delay (Fig. $6 \mathrm{~b}$ ), and $Z_{0}$ to $70 \Omega$ for $50 \Omega$ Bloch impedance (Fig. 6c). The complete RF signal path is simulated using a $2 \mathrm{D}$ electromagnetic simulator. Then the multiple-port s-parameter data is imported into a circuit simulator to simulate with transistor models.

\section{Simulation Results}

The simulated frequency response of each tap is shown in Fig. 7. The bandwidth of all five taps satisfy the cutoff frequency specification $(7 \mathrm{GHz})$, and it reduces gradually along the cells due to the larger loss associated with longer signal path. Note that the magnitude within the passband is about $-8 \mathrm{~dB}$ because of the $0.2 \mathrm{pF}$ coupling capacitor $C_{c}$.

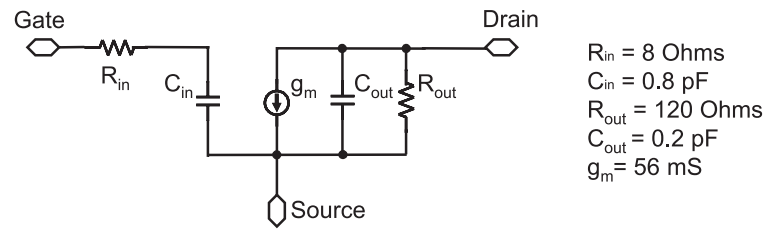

Fig. 4. Extracted transistor small-signal parameters at $V_{G S}=-0.2 \mathrm{~V}$, $V_{D S}=1.5 \mathrm{~V}, I_{D}=10.6 \mathrm{~mA}$.

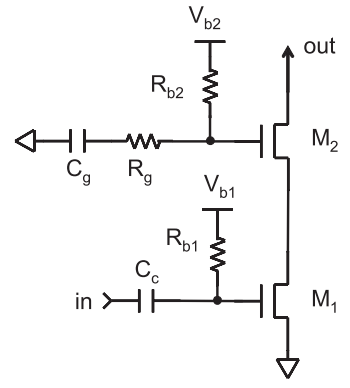

Fig. 5. Circuit schematic of the gain cell.
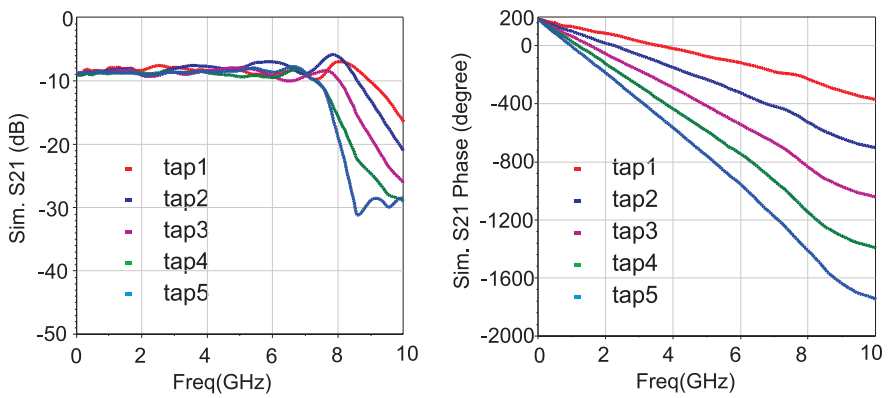

Fig. 7. Simulated frequency response of each tap.

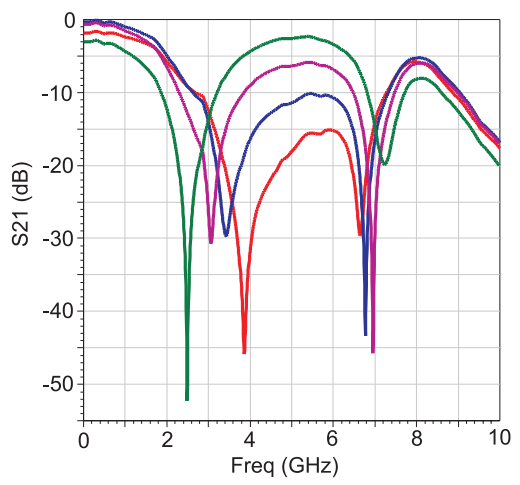

\begin{tabular}{|c|c|c|c|}
\hline Notch frequency & $g_{m 2}(\mathrm{mS})$ & $g_{m 3}(\mathrm{mS})$ & $g_{m 4}(\mathrm{mS})$ \\
\hline $2.5 \mathrm{GHz}$ & 69.0 & 5.0 & 69.0 \\
$3.0 \mathrm{GHz}$ & 69.0 & 42.0 & 69.0 \\
$3.5 \mathrm{GHz}$ & 59.1 & 69.0 & 59.1 \\
$4.0 \mathrm{GHz}$ & 42.6 & 69.0 & 42.6 \\
\hline
\end{tabular}

Fig. 8. Simulated frequency response using multiple taps. 


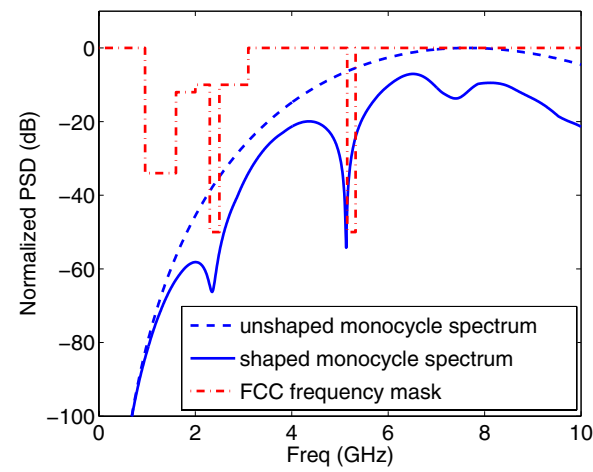

Fig. 9. Generated UWB spectrum using a monocycle input pulse (simulated). The tap gains are $g_{m 1}=0, g_{m 2}=59 \mathrm{mS}, g_{m 3}=38.5 \mathrm{mS}, g_{m 4}=38.5 \mathrm{mS}$, $g_{m 5}=59 \mathrm{mS}$

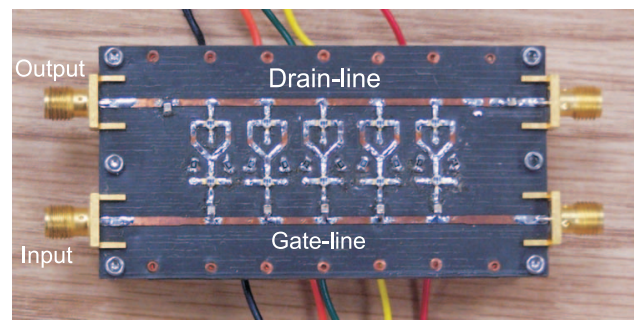

Fig. 10. Fabricated PCB prototype.

The reconfigurability is demonstrated in Fig. 8. By varying the gain of gain cell 2,3 and 4, the first notch frequency can be tuned from $2.5 \mathrm{GHz}$ to $4.0 \mathrm{GHz}$ continuously. Note that the second notch is the image of the first one around the half sampling frequency $\left(f_{s} / 2=5 \mathrm{GHz}\right)$. Fig. 9 shows the spectrum of a UWB monocycle before and after pulse shaping. The filter introduces two notches at the WLAN frequencies $(2.4 \mathrm{GHz}$ and $5.2 \mathrm{GHz}$ ) in compliance with the FCC UWB emission mask[11].

The power supply voltage is $3 \mathrm{~V}$. The current consumption depends on the tap coefficients to be generated. As an example, it consumes totally $33 \mathrm{~mA}$ to generate the spectrum in Fig. 9.

\section{Vi. Prototype Fabrication and Measurement}

The PCB prototype of the DTF is fabricated on a 20milthick Duroid5880 substrate with $\epsilon_{r}=2.2$ (Fig. 10). The
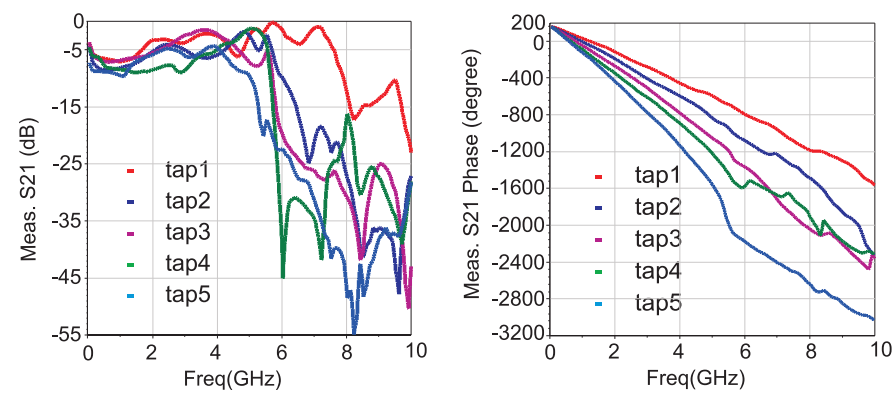

Fig. 11. Measured frequency response of each tap. measured frequency response of each tap is shown in Fig. 11. The passband gain is $-6 \mathrm{~dB}$ to $-8 \mathrm{~dB}$, close to the simulation values. The phase responses are quite linear within $5 \mathrm{GHz}$ range. The $3 \mathrm{~dB}$ bandwidth is about 4 to $5 \mathrm{GHz}$ for different taps. Compared to simulation results, the bandwidth is reduced and it exhibits larger ripples within the passband. This is largely due to parasitic effects of discrete resistors and capacitors at multi-gigahertz frequency. Another culprit is the frequency-dependent output impedance of gain cells, which is not accurately modeled in the device model. We expect to alleviate this problem in the new prototypes under construction.

\section{CONCLUSION}

We investigated the use of reconfigurable distributed transversal filters (DTFs) for multi-gigahertz pulse shaping applications. Analysis based on transmission line theory and detailed design procedures are presented. A 5-tap prototype was designed and fabricated using microwave substrate and pHEMT discrete devices. The simulation and measurement results demonstrate its pulse shaping capability and reconfigurability. We envision that this technique can be applied in a wide range of high-speed and wideband applications.

\section{ACKNOWLEDGMENT}

The authors would like to thank W. Bittle, K. Ebbecke, R. Peck and J. Henderson for their help on PCB fabrication. This work was supported by the U.S. Department of Energy Office of Inertial Confinement Fusion under Cooperative Agreement No. DE-FC52-92SF19460, the University of Rochester, and the New York State Energy Research and Development Authority. The support of DOE does not constitute an endorsement by DOE of the views expressed in this article.

\section{REFERENCES}

[1] IEEE 802.15 high rate alternative PHY task group (TG3a) merged proposal-1. http://www.uwbforum.org, 2005.

[2] UWB PHY proposal for IEEE 802.15.4a alt-PHY project. http://www.ieee802.org/15/pub/TG4a.html, 2005.

[3] H. Wu, J. Tierno, P. Pepeljugoski, J. Schaub, S. Gowda, J. Kash, and A. Hajimiri. Integrated transversal equalizers in high-speed fiber-optic systems. IEEE J. Solid-State Circuits, 38(12):2131-2137, Dec. 2003.

[4] A. Borjak, P.P. Monteiro, J.J. O'Reilly, and I. Darwazeh. Highspeed generalized distributed-amplifier-based transversal-filter topology for optical communication systems. IEEE Trans. Microwave Theory Tech., 45(8):1453-1457, Aug. 1997.

[5] Y. Zhu, J.D. Zuegel, J.R. Marciante, and H. Wu. A $0.18 \mu \mathrm{m}$ CMOS Distributed Transversal Filter for Sub-Nanosecond Pulse Synthesis. In Proc. IEEE Radio and Wireless Symp., pages 563-566, 2006.

[6] W. Jutzi. Microwave Bandwidth Active Transversal Filter Concept with MESFETs. IEEE Trans. Microwave Theory Tech., 19(9):760-767, Sep. 1971.

[7] C. Rauscher. Microwave active filters based on transversal and recursive principles. IEEE Trans. Microwave Theory Tech., 33(12):1350-1360, Dec. 1985.

[8] E.L. Ginzton, W.R. Hewlett, J.H. Jasberg, and J.D. Noe. Distributed amplification. Proc. IRE, 36:956-969, Aug. 1948.

[9] D.M. Pozar. Microwave Engineering. Wiley, 2nd edition, 1998.

[10] R.E. Collin. Foundations for Microwave Engineering. IEEE, 2nd edition, 2001.

[11] Y. Wu, A.F. Molisch, S.-Y. Kung, and J. Zhang. Impulse radio pulse shaping for ultra-wide bandwidth (uwb) systems. In IEEE Int Symposium Personal Indoor Mobile Radio Comm., volume 1, pages 877-881, 2003. 